\title{
Technical note: Characterization of ceramide in bovine lipoproteins
}

\author{
Z. C. Phipps, ${ }^{1}$ F. Seck, ${ }^{1}$ A. N. Davis, J. E. Rico, and J. W. McFadden ${ }^{2,3}$ \\ Division of Animal and Nutritional Sciences, West Virginia University, Morgantown 26505
}

\begin{abstract}
The hepatic synthesis and export of ceramide is enhanced in diabetic monogastrics. Moreover, ceramide in lipoproteins can mediate the development of insulin resistance. We have previously demonstrated that circulating ceramide increases during the progression of insulin resistance in postpartum dairy cows. Considering that the origins of circulating ceramide required investigation, our objective was to develop a method to characterize the ceramide profile of lipoprotein fractions collected from dairy cows. Serum was collected from 4 nonpregnant and nonlactating Holstein dairy cows. Serum lipoproteins were isolated using size exclusion chromatography by fast protein liquid chromatography (SEC-FPLC). Measurement of triacylglycerol (TAG), phospholipid, total cholesterol, and protein was performed using standard colorimetry practices. Following lipid extraction, fractions were analyzed using electrospray ionization tandem mass spectrometry. Data were analyzed as repeated measures using a mixed model. Lipoprotein isolation using SEC-FPLC and subsequent colorimetric analyses confirmed the presence of 4 distinct fractions: TAG-rich, low density (LDL), and large (buoyant) and small (dense) high density lipoprotein (HDL) subclasses. As expected, the fraction representing mixed very low density lipoproteins and chylomicrons primarily contained TAG. Low density lipoprotein fractions were equally enriched with cholesterol and phospholipid. Buoyant HDL contained elevated levels of cholesterol, phospholipid, and protein. In contrast, the fraction containing dense HDL primarily contained protein. Our method revealed that LDL are enriched with ceramides. Ceramides were also compartmentalized to a lesser extent within both HDL subclasses and TAG-rich lipoproteins. Comparable to whole serum, C16:0-ceramide was the predominant ceramide quantified in all lipoprotein subclasses. In-
\end{abstract}

\footnotetext{
Received December 30, 2016.

Accepted May 29, 2017.

${ }^{1}$ These authors contributed equally.

${ }^{2}$ Corresponding author: jwm43@cornell.edu

${ }^{3}$ Current address: Department of Animal Science, Cornell University, Ithaca, NY 14853.
}

terestingly, the proportion of $\mathrm{C} 24$ :0-ceramide to total ceramide was elevated in TAG-rich lipoproteins, relative to all other lipoprotein subclasses. We conclude that SEC-FPLC coupled with mass spectrometry is a means to quantify ceramides in lipoprotein fractions. Moreover, ceramides are enriched within bovine LDL, and lipoprotein ceramide profiles reflect levels observed in whole serum. Future investigation will need to determine the biological importance of lipoprotein ceramides with distinct C-chains at amide residues.

Key words: ceramide, chromatography, lipoprotein, mass spectrometry

\section{Technical Note}

The development of insulin resistance is characterized by an increase in hepatic SFA influx and the de novo synthesis of ceramide (Chavez and Summers, 2012; Pagadala et al., 2012). Ceramide can be packaged within lipoproteins and exported into systemic circulation. Additionally, ceramide accrual within low density lipoproteins (LDL) or small high density lipoproteins (dense; HDL) develops in individuals with type 2 diabetes (Boon et al., 2013; Ståhlman et al., 2013). Although the mechanisms are still unclear, current evidence suggests that LDL containing C16:0- and C24:0-ceramide can mediate the progression of insulin resistance by interfering with protein kinase B-dependent insulin signaling (Boon et al., 2013). We have recently observed an increase in circulating ceramide as cows transition from gestation to lactation, a response that occurs concomitant with fatty acid (FA) mobilization (Rico et al., 2015, 2017). Furthermore, we have demonstrated that feeding dairy cattle palmitic acid can increase plasma ceramide accumulation in association with reduced FA responsiveness to a glucose challenge (Rico et al., 2016). Considering that circulating ceramide may inhibit insulin action in dairy cows, the characterization of ceramide within lipoproteins deserves further evaluation; however, an established method is required. Therefore, our objective was to develop a technique to isolate and quantify ceramides found within bovine lipoproteins. Specifically, we applied size exclusion chromatography by fast protein liquid chromatography 
(SEC-FPLC) to isolate bovine serum lipoproteins for ceramide quantification by electrospray ionization tandem mass spectrometry (ESI-MS/MS), analytical approaches that are routinely used to evaluate lipoprotein ceramide composition in nonruminants (Wiesner et al., 2009; Kontush and Chapman, 2010; Boon et al., 2013).

Experimental procedures were approved by the Institutional Animal Care and Use Committee at West Virginia University (Morgantown). To characterize the ceramide profile of lipoproteins, blood samples were collected from 4 nonpregnant, nonlactating Holstein cows (35 to 48 mo of age) housed in individual strawbedded pens at the West Virginia University Animal Science Farm. Cows were fed a TMR composed of grass hay and ground corn formulated to meet nutrient recommendations (Supplemental Table S1; https:/ / doi.org/10.3168/jds.2016-12538). Cows were fed twice daily (0900 and $1700 \mathrm{~h})$ at $110 \%$ of expected intake and provided free access to water at all times. The mixed ration was collected and stored at $-20^{\circ} \mathrm{C}$. Basal blood collection $(10 \mathrm{~mL})$ from a jugular vein occurred at 0600 h. Blood was kept on ice for 30 min until centrifugation at $3,400 \times g$ for $10 \mathrm{~min}$ at $4^{\circ} \mathrm{C}$. Because lipoprotein fractionation does not appear to be influenced by freezing (Nanjee and Brinton, 2000; Wiesner et al., 2009; Collins et al., 2010), serum was stored at $-80^{\circ} \mathrm{C}$ until SEC-FPLC injection.

Individually composited feed ingredients were analyzed for NDF with heat-stable $\alpha$-amylase and sodium sulfite (Van Soest et al., 1991), CP (AOAC International, 2000; method 990.03), and starch (Hall, 2009) by Cumberland Valley Analytical Services Inc. (Cumberland, MD).

Whole serum samples and SEC-FPLC fractions were analyzed in duplicate for triacylglycerol (TAG; kit \#T7532; Pointe Scientific Inc., Canton, MI), total cholesterol and phospholipid using enzymatic methods [Cholesterol E (kit \#999-02601) and Phospholipid C (kit \#997-01801), respectively; Wako Chemicals USA Inc., Richmond, VA], and total protein using a bicinchoninic acid assay (kit \#23225, Thermo Scientific, Hudson, NH). Whole serum FA and glucose levels were also determined [HR series NEFA-HR (kit \#999-34691) and Autokit glucose (kit \#997-03001), respectively; Wako Chemicals USA Inc.]. Spectrophotometric measurements were conducted using a SpectraMax Plus 384 Microplate Reader (Molecular Devices, Sunnyvale, $\mathrm{CA})$. Intra- and interassay coefficients of variation were 2.8 and $2.9 \%, 2.2$ and $3.4 \%, 1.7$ and $9.8 \%$, and 3.7 and $6.1 \%$ for TAG, total cholesterol, phospholipids, and protein, respectively. The intraassay coefficients of variation for serum FA and glucose were 3.2 and $3.6 \%$, respectively.
Lipoprotein isolation was performed using a BioLogic DuoFlow 10 System equipped with a BioFrac fraction collector (Bio-Rad Laboratories, Hercules, CA) following previously established methods (Collins et al., 2010). Analytical SEC-FPLC was carried out on a single Superdex $65010 \times 300 \mathrm{~mm}$ column (Bio-Rad Laboratories). Elutions were performed in a $1 \mathrm{mM}$ EDTA, $150 \mathrm{mM} \mathrm{NaCl}, 0.02 \% \mathrm{NaN}_{3}$ phosphate-buffered solution, $\mathrm{pH}$ 7.4. This eluent buffer was degassed in a strong-side Erlenmeyer flask for 30 min under vacuum. The eluent buffer was also sterile filtered through a 0.1 $\mu \mathrm{m}$ vacuum-driven GP Express PLUS membrane (Millipore, Billerica, MA). Prior to each injection into the SEC-FPLC system, $12 \mathrm{~mL}$ of eluent buffer $(0.3 \mathrm{~mL} /$ min) was used to ensure equilibration of the column. The column was washed with degassed and filtered eluent buffer between runs. Immediately before injection, single thawed serum samples were centrifuged at 10,000 $\times g$ for $5 \mathrm{~min}$. Serum sample aliquots of $350 \mu \mathrm{L}$ were used for each injection. To completely fill the injection loop, 1,000 $\mu \mathrm{L}$ of eluent buffer was also added to each injection. The elutions were carried out at a flow rate of $0.3 \mathrm{~mL} / \mathrm{min}$ with a maximum pressure of 820 $\mathrm{kPa}$ and continually monitored at an absorbance of 280 $\mathrm{nm}$. Individual fraction collection $(0.5 \mathrm{~mL})$ was initiated $17 \mathrm{~min}, 50 \mathrm{~s}$ after sample injection and presented as volume collected for analysis. To prevent sample adhesiveness to the polypropylene fraction collection tubes, the tubes were pretreated with a $0.1 \%$ Tween 20 (Sigma-Aldrich, St. Louis, MO) solution as described by Nanjee and Brinton (2000). Fraction volumes corresponding to discrete elution peaks or troughs (or both) were pooled and saved for subsequent analysis.

Whole serum and pooled SEC-FPLC fractions were extracted using a modified Bligh and Dyer procedure using C12:0-ceramide as an internal standard (Avanti Polar Lipids, Alabaster, AL). A previously established method was employed (Haughey et al., 2004; Rico et al., 2015, 2016); however, a larger SEC-FPLC fraction volume $(350 \mu \mathrm{L})$ was required. Following extraction with proportional volumes of organic solvent, the extracts were concentrated under nitrogen gas (Organomation Associates Inc., Berlin, MA) and re-suspended in pure methanol before analysis. Ceramides were separated by gradient elution using UHPLC (ExionLC AD, SCIEX, Framingham, MA) on a $\mathrm{C} 18$ reverse-phase column (Phenomenex, Torrance, CA). Ceramides were detected using ESI-MS/MS as previously described (QTRAP 5500, SCIEX; Mielke et al., 2015). Ionspray source voltage was $5,000 \mathrm{~V}$ at a temperature of $500^{\circ} \mathrm{C}$, and a nebulizer, heater, curtain, and collision gas pressure of $483,414,193$, and $62 \mathrm{kPa}$, respectively. For ceramide optimization parameters, declustering potential was 30 
to $50 \mathrm{~V}$, entrance potential 10 to $15 \mathrm{~V}$, collision energy 32 to $37 \mathrm{~V}$, and collision cell exit potential 13 to $17 \mathrm{~V}$. Individual ceramide species were detected by multiple reaction monitoring of the $[\mathrm{M}+\mathrm{H}]^{+}$molecular ion with transition ion of $264.2 \mathrm{~m} / \mathrm{z}$. A 10-point calibration curve $(0.5$ to $500 \mathrm{ng} / \mathrm{mL}$ ) was constructed by plotting the area under the curve for C16:0-, C18:0-, C22:0-, and C24:0-ceramide (Avanti Polar Lipids). Fluctuations in extraction and mass spectrometer efficiencies were controlled by C12:0-ceramide normalization and samples were re-run if the internal standard response deviated more than $10 \%$ from its median value. Ceramide concentrations were determined by curve-fitting the identified ceramide species based on the chain length of the fatty acid at the amide bond. Samples were injected in duplicate to ensure similar response (the overall mean CV was $6.54 \% ; \mathrm{R}^{2}$ values for all standard curves were 0.99). Instrument control and quantitation were performed using Analyst 1.6.3 software (SCIEX).

All data were analyzed as repeated measures using the MIXED model procedure of SAS (version 9.3, SAS Institute Inc., Cary, NC).

$$
\mathrm{Y}_{\mathrm{ijk}}=\mu+\mathrm{C}_{\mathrm{i}}+\mathrm{P}_{\mathrm{j}}+\mathrm{e}_{\mathrm{ijk}},
$$

where $\mathrm{Y}_{\mathrm{ijk}}=$ dependent variable, $\mu=$ overall mean, $\mathrm{C}_{\mathrm{i}}$ $=$ random effect of cow ( $\mathrm{i}=1$ to 4$), \mathrm{P}_{\mathrm{j}}=$ fixed effect of individual or pooled SEC-FPLC fraction $(\mathrm{j}=1$ to 30 or $\mathrm{j}=1$ to 4 ), and $\mathrm{e}_{\mathrm{ijk}}=$ residual error. Normality of the residuals was checked with normal probability and box plots, and homogeneity of variances with plots of residual versus predicted values. Significance was declared at $P<0.05$. All results are expressed as least squares means and their standard errors unless stated otherwise.

The 4 cows were diagnosed as clinically healthy at the time of sampling. Dry matter intake averaged 18.6 $\pm 1.1 \mathrm{~kg} / \mathrm{d}$ the week before blood collection. Serum concentrations of FA $(72.0 \pm 13.4 \mu \mathrm{mol} / \mathrm{L})$, glucose $(49.0 \pm 12.8 \mathrm{mg} / \mathrm{dL})$, TAG $(17.0 \pm 1.9 \mathrm{mg} / \mathrm{dL})$, total cholesterol $(133 \pm 21 \mathrm{mg} / \mathrm{dL})$, phospholipid (141 \pm $11 \mathrm{mg} / \mathrm{dL})$, and protein $(102 \pm 11 \mathrm{mg} / \mathrm{dL})$ reflected a healthy metabolic state.

In the laboratory, we evaluated the reproducibility of the SEC-FPLC fractionation by examining 350- $\mu \mathrm{L}$ aliquots of whole bovine serum collected from all 4 cows (Figure 1A). Fractionated over the same SEC column, we observed that elution profiles are reproducible, demonstrating that different lipoprotein classes elute at fixed times. To provide molecular weight estimates of lipoprotein classes, we individually loaded thyroglobulin (669 kDa), apoferritin (443 kDa), serum albumin (67 $\mathrm{kDa})$, and carbonic anhydrase $(29 \mathrm{kDa})$ onto and chro-
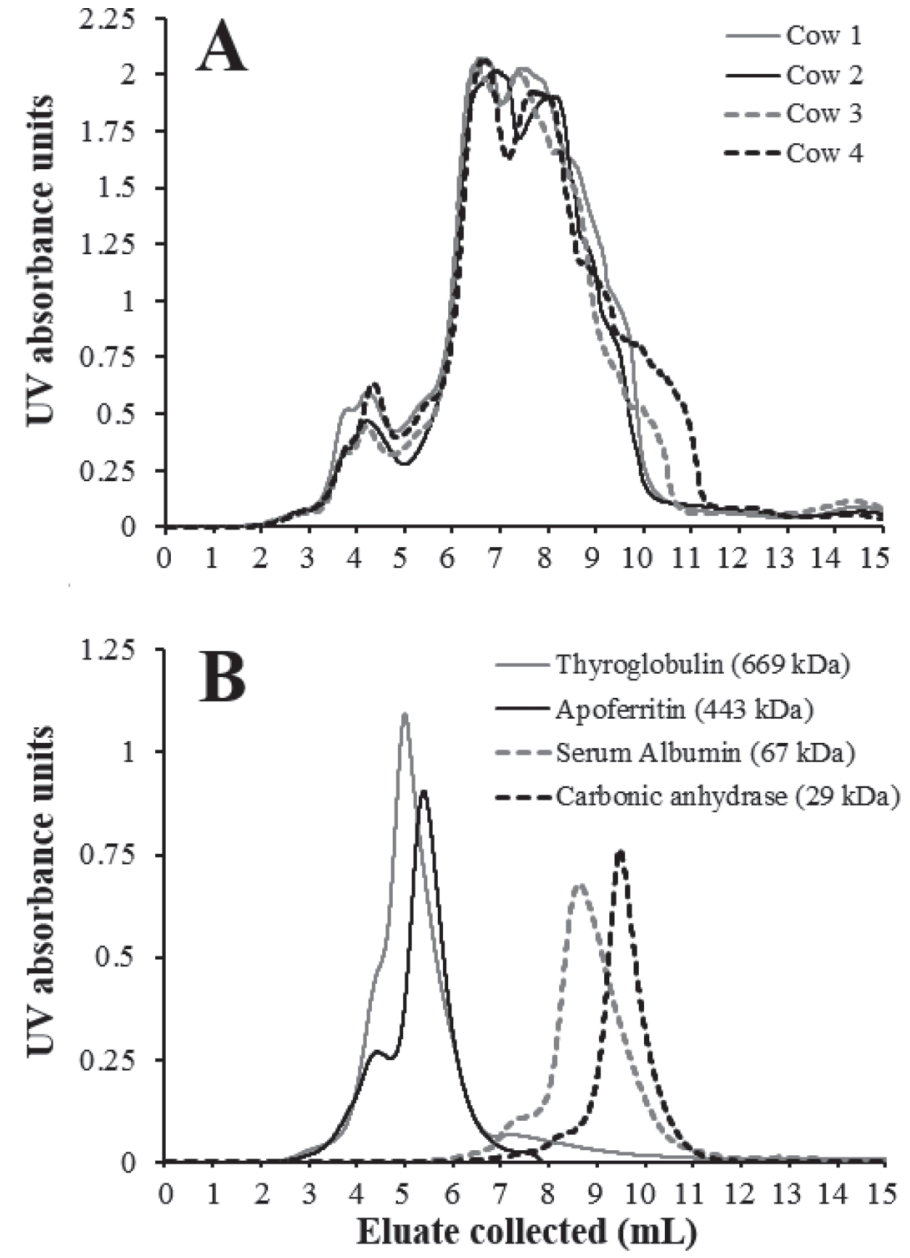

Figure 1. Fast protein liquid chromatography elution profiles. (A) Size exclusion elution profiles obtained using fast protein liquid chromatography. Each elution profile was obtained following a $350-\mu \mathrm{L}$ injection of bovine serum from 4 cows. (B) Individual elution profiles of protein molecular weight standards. Volumes represent fractions collected $17 \mathrm{~min}, 50 \mathrm{~s}$ after sample injection.

matographed over the SEC column (Figure 1B). The elution volumes of apoferritin and carbonic anhydrase flank the bifurcated major peak localized between 6.0 and $9.5 \mathrm{~mL}$ of eluate (Figure 1A), denoted by Collins et al. (2010) as representative of large (buoyant) and dense HDL subclasses.

We next employed colorimetric spectrophotometry to characterize the concentrations of TAG, total cholesterol, phospholipid, and protein of each $0.5-\mathrm{mL}$ lipoprotein fraction (Figure 2). Comparable to the recorded UV absorbance, protein enrichment developed between 6.5 and $10.5 \mathrm{~mL}$ of eluate with the greatest concentration of protein measured between 7.0 and $9.0 \mathrm{~mL}$ of eluate. The concentration of TAG was maximum at $3.5 \mathrm{~mL}$ of eluate, preceding the thyroglobulin protein marker. We observed a $72 \%$ decrease in circulating TAG concentra- 
Table 1. Major lipid and protein composition of lipoprotein fractions

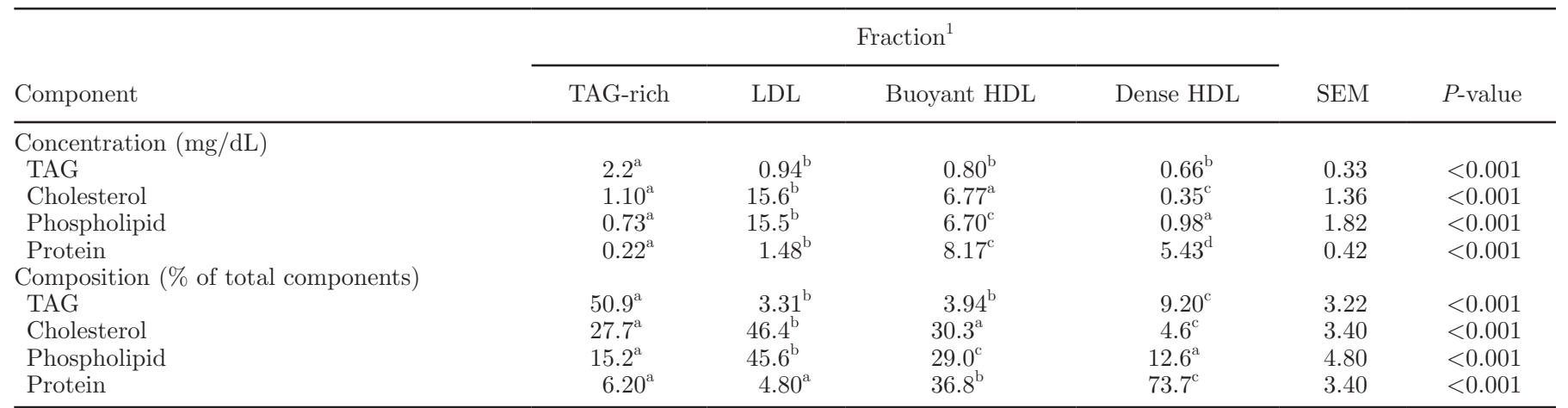

${ }^{\mathrm{a}-\mathrm{d}}$ Differing superscript letters indicate statistically significant difference within component $(P<0.05)$.

${ }^{1}$ Pooled fractions collected using fast protein liquid chromatography represent triacylglycerol (TAG)-rich (2.5 to $\left.4.0 \mathrm{~mL}\right)$, low density (5.0 to $6.5 \mathrm{~mL}$; LDL), and high density large (7.0 to $8.5 \mathrm{~mL}$; buoyant HDL) and small (8.5 to $10.0 \mathrm{~mL}$; dense HDL) lipoprotein subclasses. Volumes represent fractions collected $17 \mathrm{~min}, 50 \mathrm{~s}$ after sample injection.

tion from 3.5 to $5.0 \mathrm{~mL}$ of eluate, and TAG remained low beyond $5.0 \mathrm{~mL}$ of eluted sample. In contrast, the concentrations of cholesterol and phospholipid were most pronounced between 4.0 and $9.0 \mathrm{~mL}$ (peaked at $6.0 \mathrm{~mL}$ ). Considering these observations, SEC-FPLC fractions corresponding to discrete elution peaks or troughs (or both) were pooled for subsequent ESI-MS/ MS analysis. Specifically, we pooled 2.5 to $4.0 \mathrm{~mL}, 5.0$ to $6.5 \mathrm{~mL}, 7.0$ to $8.5 \mathrm{~mL}$, and 8.5 to $10.0 \mathrm{~mL}$ to reflect the TAG-rich [very low density lipoproteins (VLDL) and chylomicrons], LDL, and buoyant and dense HDL lipoprotein subclasses, respectively. The concentrations of TAG, total cholesterol, phospholipid, and protein in pooled SEC-FPLC fractions is detailed within Table 1. Unlike TAG-rich lipoprotein particles, the TAG content of the pooled SEC-FPLC fractions representing LDL was minimal. In contrast, equal enrichment of cholesterol and phospholipid within LDL fractions was observed. We also quantified the lipid and protein content of buoyant and dense HDL fraction sets. An equal proportion of cholesterol, phospholipid, and protein within the buoyant HDL fractions was detected; however, the

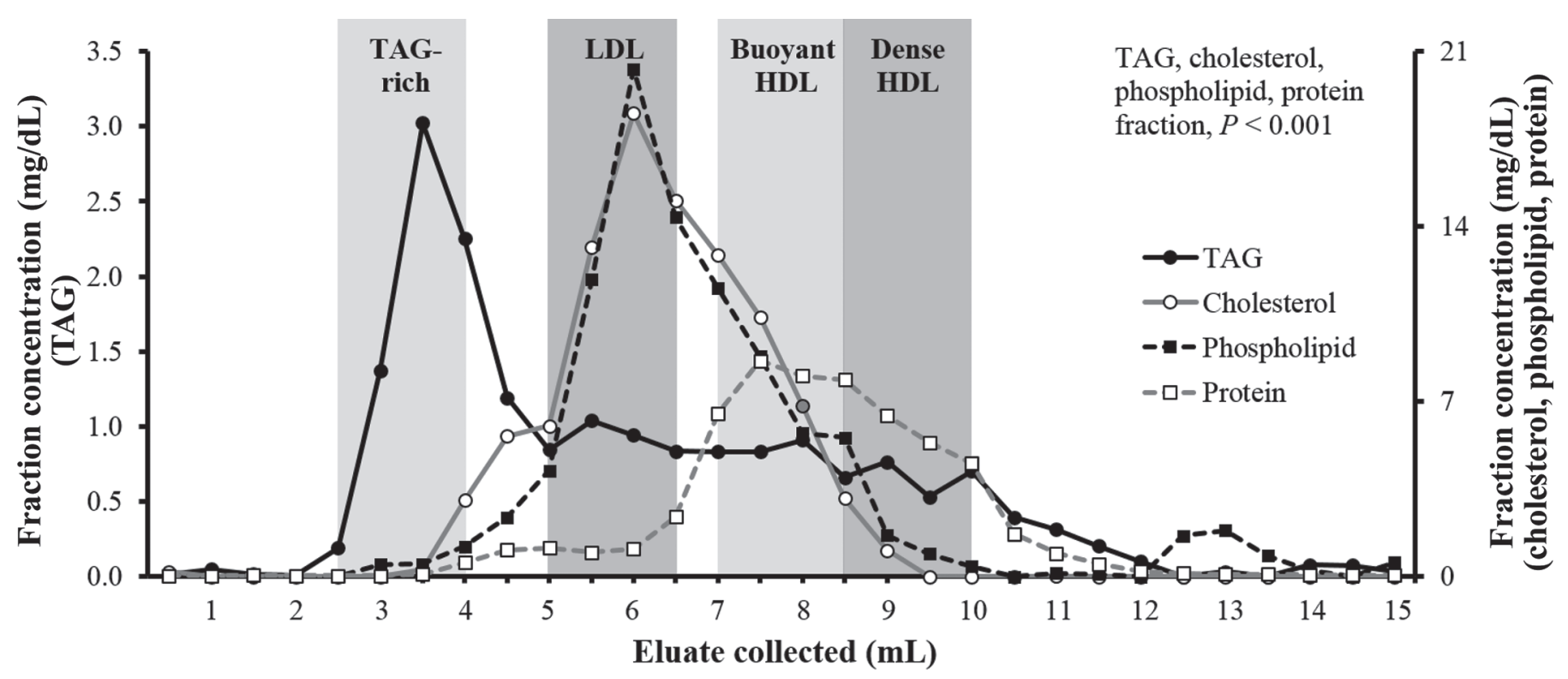

Figure 2. Major lipid and protein composition of serum lipoprotein fractions. Size exclusion fractions were collected using fast protein liquid chromatography following a 350- $\mu \mathrm{L}$ injection of serum collected from 4 nonpregnant and nonlactating Holstein dairy cows. Following fractionation, colorimetric analysis of triacylglycerol (TAG), total cholesterol, phospholipid, and protein concentrations of fractions was performed. The shaded regions denote TAG-rich $(2.5$ to $4.0 \mathrm{~mL}$ ), low density (5.0 to $6.5 \mathrm{~mL}$; LDL), and high density large (7.0 to $8.5 \mathrm{~mL}$; buoyant HDL) and small ( 8.5 to $10.0 \mathrm{~mL}$; dense HDL) lipoprotein subclasses. Volumes represent fractions collected 17 min, $50 \mathrm{~s}$ after sample injection. Data are represented as LSM. The overall SEM equals $0.26,0.98,1.38$, and 0.35 for TAG, total cholesterol, phospholipid, and protein, respectively. 
dense HDL fraction was primarily composed of protein. Because these results reflected our expectations and confirm earlier findings in dairy cows (Raphael et al., 1973; Stead and Welch, 1975; Grummer and Davis, 1984), the ceramide profile of each lipoprotein subclass was characterized.

We used ESI-MS/MS to quantify C16:0-, C18:0-, C18:1-, C22:0-, C24:0-, and C24:1-ceramide concentrations within whole serum and pooled chromatography fractions representing TAG-rich lipoprotein particles (VLDL and chylomicrons), LDL, and buoyant and dense HDL (Table 2). Noticeably, the LDL fraction contained the greatest concentration of total ceramide. To a lesser extent, ceramide was also quantified within buoyant and dense HDL fractions. Ceramide levels within the TAG-rich fraction were low. Comparable to whole serum, bovine lipoproteins primarily contained C16:0- and C24:0-ceramide. Moreover, the composition of ceramide within LDL, and buoyant and dense HDL was comparable (e.g., C16:0- and C24:0-ceramide represented $76-86 \%$ and $7.6-8.8 \%$ of total ceramide, respectively). In sharp contrast, C16:0- and C24:0 -ceramide represented 38.9 and $40.1 \%$ of total ceramide within the TAG-rich fraction.

The characterization of lipoprotein TAG, cholesterol, phospholipid, and protein composition in dairy cows has been extensively characterized (Raphael et al., 1973; Stead and Welch, 1975; Bauchart, 1993). Researchers have previously applied ultracentrifugation and gel filtration chromatography techniques to demonstrate enrichment of TAG within bovine VLDL and chylomicrons, equivalent concentrations of total cholesterol and phospholipid within LDL and buoyant HDL, and minimal lipid content within dense HDL (Raphael et al., 1973; Oikawa et al., 2010). In our investigation, a SEC-FPLC approach revealed a similar chemical composition for these lipoprotein subclasses. Furthermore, our approach generated a SEC-FPLC elution profile (UV absorption spectra and protein marker elution) that is comparable to previous work involving human lipoprotein fractionation (Collins et al., 2010). We selected a SEC-FPLC application because ultracentrifugal methods require large volumes of samples, lengthy run times, and high centrifugal forces and salt concentrations that may cause dissociation of lipoproteins (Kunitake and Kane, 1982). Alternatively, our SEC-FPLC method required a smaller sample volume, shorter run time, and minimized lipoprotein denaturation as a means of isolating lipoproteins for mass spectrometry-based lipidomics (Wiesner et al., 2009; Collins et al., 2010). The inability to distinguish chylomicrons from hepatic-derived VLDL represents a potential limitation of these approaches when blood is collected in the fed state, a constraint that could be overcome by sampling in the fasted state or directly from the hepatic vein. Additionally, future application of this method should attempt to detect apolipoproteins that are specific for each lipoprotein subclass (e.g., VLDL apolipoprotein $\mathrm{B}_{100}$ ).

Circulating ceramide is formed de novo from SFA in part by the actions of hepatic serine palmitoyltransferase and ceramide synthase. Alternatively, tissue

Table 2. Ceramide concentration and composition of whole serum and lipoprotein fractions

\begin{tabular}{|c|c|c|c|c|c|c|c|}
\hline Ceramide $^{1}$ & $\begin{array}{l}\text { Whole } \\
\text { serum }\end{array}$ & \multicolumn{4}{|c|}{ Fraction $^{2}$} & SEM & $P$-value \\
\hline \multicolumn{8}{|c|}{ Concentration $(\mathrm{ng} / \mathrm{mL})$} \\
\hline C18:0 & 3.66 & $\mathrm{ND}^{3}$ & 1.24 & 0.63 & 0.50 & 0.49 & 0.22 \\
\hline $\mathrm{C} 18: 1$ & 3.82 & $0.63^{\mathrm{a}}$ & $2.11^{\mathrm{b}}$ & $1.30^{\mathrm{a}}$ & $1.34^{\mathrm{ab}}$ & 0.33 & 0.02 \\
\hline $\mathrm{C} 22: 0$ & 12.9 & $1.22^{\mathrm{a}}$ & $1.31^{\mathrm{a}}$ & $0.52^{\mathrm{b}}$ & $0.51^{\mathrm{ab}}$ & 0.47 & 0.08 \\
\hline \multicolumn{8}{|c|}{ Composition ( $\%$ of total ceramides) } \\
\hline C16:0 & 49.4 & $38.9^{\mathrm{a}}$ & $75.6^{\mathrm{b}}$ & $80.1^{\mathrm{bc}}$ & $85.9^{c}$ & 3.53 & $<0.001$ \\
\hline C18:0 & 3.4 & ND & 3.27 & 2.79 & 2.35 & 0.47 & 0.24 \\
\hline C18:1 & 3.5 & $4.68^{\mathrm{a}}$ & $5.74^{\mathrm{b}}$ & $6.68^{\mathrm{c}}$ & $6.81^{\mathrm{c}}$ & 0.35 & $<0.01$ \\
\hline $\mathrm{C} 22: 0$ & 11.9 & $14.8^{\mathrm{a}}$ & $3.52^{\mathrm{b}}$ & $2.75^{\mathrm{b}}$ & $1.81^{\mathrm{b}}$ & 1.98 & $<0.001$ \\
\hline C24:0 & 25.0 & $40.1^{\mathrm{a}}$ & $8.81^{b}$ & $7.82^{\mathrm{b}}$ & $7.60^{\mathrm{b}}$ & 3.73 & $<0.001$ \\
\hline
\end{tabular}

\footnotetext{
${ }^{\mathrm{a}-\mathrm{c}}$ Differing superscript letters indicate statistically significant difference within component $(P<0.05)$.

${ }^{1}$ Ceramide composition represents percentage of total ceramide detected.

${ }^{2}$ Pooled fractions collected using fast protein liquid chromatography represent triacylglycerol (TAG)-rich (2.5 to $4.0 \mathrm{~mL}$ ), low density (5.0 to $6.5 \mathrm{~mL}$; LDL), and high density large (7.0 to $8.5 \mathrm{~mL}$; buoyant HDL) and small ( 8.5 to $10.0 \mathrm{~mL}$; dense HDL) lipoprotein subclasses. Volumes represent fractions collected $17 \mathrm{~min}, 50 \mathrm{~s}$ after sample injection.

${ }^{3} \mathrm{ND}=$ not detected.
} 
or LDL-associated sphingomyelinase can hydrolyze sphingomyelin to form ceramide (Holopainen et al., 2000). In our investigation, ceramide enrichment within SEC-FPLC fractions containing LDL was observed. Previous efforts have confirmed that $60 \%$ of lipoprotein ceramides are found within serum LDL fractions collected using FPLC and blood collected from fasted human donors (Wiesner et al., 2009). In addition to LDL, the current study revealed that ceramide accumulation occurs within buoyant and dense HDL. Prior findings have demonstrated that HDL represents the second major lipoprotein compartment for ceramide accrual ( $\sim 24-45 \%$ of lipoprotein ceramide; Wiesner et al., 2009; Boon et al., 2013), and buoyant HDL exhibit a higher ceramide content relative to dense HDL (Camont et al., 2013). To a far less extent, we detected ceramide within TAG-rich lipoprotein particles. Increasing hepatic FA supply enhances ceramide synthesis and export as part of nascent VLDL (Merrill et al., 1995; Watt et al., 2012). In the present study, the observed cows were clinically healthy and FA supply to the liver was minimal; therefore, hepatic ceramide synthesis and export as VLDL may have been limited. The contribution of ceramide within the TAG-rich fraction is likely hepaticderived VLDL and not intestinal chylomicrons because the amide bond of dietary ceramide is hydrolyzed by intestinal ceramidase (Olsson et al., 2004). Moreover, ceramide represents $0.3 \%$ of chylomicron polar surface lipids (Yang et al., 1992).

Ceramide lipoprotein compartmentalization may influence whether a particular ceramide moiety influences insulin action (Boon et al., 2013; Ståhlman et al., 2013). In dairy cattle, we have observed marked elevations in circulating very long chain ceramides as cows transition from gestation to lactation (most notably C24:0 -ceramide); however, C16:0-ceramide declines during the transition (Rico et al., 2015). These responses develop in parallel with increased adipose tissue lipolysis and reduced insulin sensitivity. Similar observations were observed in mid-lactation dairy cows fed supplemental palmitic acid (Rico et al., 2016). In the present study, C16:0-ceramide within LDL and HDL was highly abundant in nonlactating, nongestating dairy cows that were likely insulin-sensitive. In contrast, C24:0-ceramide is the most abundant ceramide in pregnant or lactating dairy cows (Rico et al., 2015, 2016); however, we acknowledge that the contribution of the C16:0 moiety to the circulating ceramide pool increases as lactation advances beyond peak milk production. Interestingly, C24:0-ceramide incorporation within TAG-rich lipoprotein particles was enhanced, relative to $\mathrm{C} 16$ :0-ceramide. We note that differences in circulating lipoprotein ceramide profiles may reflect distinct dietary regimens or physiological states. The future application of these
SEC-FPLC and ESI-MS/MS methods will enable the characterization of lipoprotein ceramide profiles during the peripartum and determine whether lipoprotein ceramide mediates insulin resistance in dairy cattle.

We conclude that a method involving SEC-FPLC and ESI-MS/MS can be employed to isolate and quantify ceramides in bovine lipoproteins. The described methods can be used to characterize other complex lipid classes using targeted and untargeted lipidomics approaches, or be applied to investigate specific apolipoproteins. We have also demonstrated that circulating ceramide is found within multiple lipoprotein subclasses but predominantly found within serum LDL collected from nonlactating, nongestating Holstein dairy cows in the basal state. Additionally, lipoprotein ceramide profiles reflect levels observed in whole serum.

\section{ACKNOWLEDGMENTS}

This project was supported by Agriculture and Food Research Initiative Competitive Grant no. 2016-6701524582 from the USDA National Institute of Food and Agriculture (Washington, DC), the West Virginia University Davis College of Agriculture, Natural Resources and Design (Morgantown), and the West Virginia University School of Medicine (Morgantown). We also gratefully acknowledge Greg Boyce (West Virginia University, Morgantown) for assisting with the mass spectrometry analysis.

\section{REFERENCES}

AOAC International. 2000. Official Methods of Analysis. 17th ed. AOAC International, Gaithersburg, MD.

Bauchart, D. 1993. Lipid absorption and transport in ruminants. J. Dairy Sci. 76:3864-3881.

Boon, J., A. J. Hoy, R. Stark, R. D. Brown, R. C. Meex, D. C. Henstridge, S. Schenk, P. J. Meikle, J. F. Horowitz, B. A. Kingwell, C. R. Bruce, and M. J. Watt. 2013. Ceramides contained in LDL are elevated in type 2 diabetes and promote inflammation and skeletal muscle insulin resistance. Diabetes 62:401-410.

Camont, L., M. Lhomme, F. Rached, W. Le Goff, A. Nègre-Salvayre, R. Salvayre, C. Calzada, M. Lagarde, M. J. Chapman, and A. Kontush. 2013. Small, dense high-density lipoprotein-3 particles are enriched in negatively charged phospholipids: Relevance to cellular cholesterol efflux, antioxidative, antithrombotic, anti-inflammatory, and antiapoptotic functionalities. Arterioscler. Thromb. Vasc. Biol. 33:2715-2723.

Chavez, J. A., and S. A. Summers. 2012. A ceramide-centric view of insulin resistance. Cell Metab. 15:585-594.

Collins, L. A., S. P. Mirza, A. H. Kissebah, and M. Olivier. 2010. Integrated approach for the comprehensive characterization of lipoproteins from human plasma using FPLC and nano-HPLC-tandem mass spectrometry. Physiol. Genomics 40:208-215.

Grummer, R. R., and C. L. Davis. 1984. Plasma concentration and lipid composition of lipoproteins in lactating dairy cows fed control and high grain diets. J. Dairy Sci. 67:2894-2901.

Hall, M. B. 2009. Determination of starch, including maltooligosaccharides, in animal feeds: Comparison of methods and a method recommended for AOAC collaborative study. J. AOAC Int. 92:42-49. 
Haughey, N. J., R. G. Cutler, A. Tamara, J. C. McArthur, D. L. Vargas, C. A. Pardo, J. Turchan, A. Nath, and M. P. Mattson. 2004 Perturbation of sphingolipid metabolism and ceramide production in HIV-dementia. Ann. Neurol. 55:257-267.

Holopainen, J. M., O. P. Medina, A. J. Metso, and P. K. J. Kinnunen. 2000. Sphingomyelinase activity associated with human plasma low density lipoprotein: Possible functional implications. J. Biol. Chem. 275:16484-16489.

Kontush, A., and M. J. Chapman. 2010. Lipidomics as a tool for the study of lipoprotein metabolism. Curr. Atheroscler. Rep. 12:194201.

Kunitake, S. T., and J. P. Kane. 1982. Factors affecting the integrity of high density lipoproteins in the ultracentrifuge. J. Lipid Res. 23:936-940.

Merrill, A. H., S. Lingrell, E. Wang, M. Nikolova-Karakashian, T. R. Vales, and D. E. Vance. 1995. Sphingolipid biosynthesis de novo by rat hepatocytes in culture. Ceramide and sphingomyelin are associated with, but not required for, very low density lipoprotein secretion. J. Biol. Chem. 270:13834-13841.

Mielke, M. M., V. V. R. Bandaru, D. Han, Y. An, S. M. Resnick, L. Ferrucci, and N. J. Haughey. 2015. Demographic and clinical variables affecting mid- to late-life trajectories of plasma ceramides and dihydroceramide species. Aging Cell 14:1014-1023.

Nanjee, M. N., and E. A. Brinton. 2000. Very small apolipoprotein A-I-containing particles from human plasma: isolation and quantification by high-performance size-exclusion chromatography. Clin. Chem. 46:207-223.

Oikawa, S., Y. Mizunuma, Y. Iwasaki, and M. Tharwat. 2010. Changes of very low-density lipoprotein concentration in hepatic blood from cows with fasting-induced hepatic lipidosis. Can. J. Vet. Res. 74:317-320.

Olsson, M., R.-D. Duan, L. Ohlsson, and A. Nilsson. 2004. Rat intestinal ceramidase: Purification, properties, and physiological relevance. Am. J. Physiol. Gastrointest. Liver Physiol. 287:G929 G937.

Pagadala, M., T. Kasumov, A. J. McCullough, N. N. Zein, and J. P. Kirwan. 2012. Role of ceramides in nonalcoholic fatty liver disease. Trends Endocrinol. Metab. 23:365-371.

Raphael, B. C., P. S. Dimick, and D. L. Puppione. 1973. Lipid characterization of bovine serum lipoproteins throughout gestation and lactation. J. Dairy Sci. 56:1025-1032.
Rico, J. E., V. V. Bandaru, J. M. Dorskind, N. J. Haughey, and J. W. McFadden. 2015. Plasma ceramides are elevated in overweight Holstein dairy cows experiencing greater lipolysis and insulin resistance during the transition from late pregnancy to early lactation. J. Dairy Sci. 98:7757-7770.

Rico, J. E., A. T. Mathews, J. Lovett, N. J. Haughey, and J. W. McFadden. 2016. Palmitic acid feeding increases ceramide supply in association with increased milk yield, circulating nonesterified fatty acids, and adipose tissue responsiveness to a glucose challenge. J. Dairy Sci. 99:8817-8830.

Rico, J. E., S. Saed Samii, A. T. Mathews, J. Lovett, N. J. Haughey and J. W. McFadden. 2017. Temporal changes in sphingolipids and systemic insulin sensitivity during the transition from gestation to lactation. PLoS One 12:e0176787.

Ståhlman, M., B. Fagerberg, M. Adiels, K. Ekroos, J. M. Chapman, A. Kontush, and J. Borén. 2013. Dyslipidemia, but not hyperglycemia and insulin resistance, is associated with marked alterations in the HDL lipidome in type 2 diabetic subjects in the DIWA cohort: Impact on small HDL particles. Biochim. Biophys. Acta 1831:1609-1617.

Stead, D., and V. A. Welch. 1975. Lipid composition of bovine serum lipoproteins. J. Dairy Sci. 58:122-127.

Van Soest, P. J., J. B. Robertson, and B. A. Lewis. 1991. Methods for dietary fiber, neutral detergent fiber, and nonstarch polysaccharides in relation to animal nutrition. J. Dairy Sci. 74:3583-3597.

Watt, M. J., A. C. Barnett, C. R. Bruce, S. Schenk, J. F. Horowitz, and A. J. Hoy. 2012. Regulation of plasma ceramide levels with fatty acid oversupply: Evidence that the liver detects and secretes de novo synthesised ceramide. Diabetologia 55:2741-2746.

Wiesner, P., K. Leidl, A. Boettcher, G. Schmitz, and G. Liebisch. 2009. Lipid profiling of FPLC-separated lipoprotein fractions by electrospray ionization tandem mass spectrometry. J. Lipid Res. 50:574-585.

Yang, L. Y., A. Kuksis, J. J. Myher, and H. Pang. 1992. Surface components of chylomicrons from rats fed glyceryl or alkyl esters of fatty acids: Minor components. Lipids 27:613-618. 